\title{
Safety and Immunogenicity of Recombinant, Live Attenuated Tetravalent Dengue Vaccine (CYD- TDV) in Healthy Vietnamese Adults and Children
}

Ngoc Huu Tran'1, Chan Quang Luong ${ }^{1}$, Thi Que Huong Vu¹, Remi Forrat ${ }^{2}$, Jean Lang ${ }^{3}$, Quoc Dat Vu ${ }^{4}$, Alain Bouckenooghe ${ }^{4}$ and Tram Anh Wartel ${ }^{5 *}$

${ }^{1}$ Ho Chi Minh City Pasteur Institute, Ho Chi Minh, Vietnam

'Sanofi Pasteur Clinical Department, Marcy L'Etoile, France

${ }^{3}$ Sanofi Pasteur R\&D, Marcy L'Etoile, France

${ }^{4}$ Sanofi Pasteur Clinical R\&D, Singapore

${ }^{5}$ Sanofi Pasteur Clinical R\&D, Bangkok, Thailand

\begin{abstract}
Background: Dengue viruses (DENV1-4) are estimated to infect 50-100 million individuals per year worldwide including an estimated 500,000 people with severe dengue who require hospitalization every year. The live, attenuated, tetravalent dengue vaccine (CYD-TDV) candidate, containing four recombinant dengue viruses (CYD14 ), is in clinical phase III.
\end{abstract}

Methods: In an observer-blind, phase II trial in Long Xuyen, Vietnam, 180 children and adults (range: 2-45 years) were randomized 2:1 to receive 3 CYD-TDV vaccinations at months (M) 0,6 and 12 or meningococcal polysaccharide $A+C$ at $M 0$, placebo at $M 6$, and typhoid Vi polysaccharide at M12. Serum antibody responses against the CYD1-4 parental wild-type dengue viruses were assessed using plaque-reduction neutralization test (PRNT50). Safety and reactogenicity were assessed using conventional methods. Febrile episodes lasting $\geq 48 \mathrm{~h}$ with suspicion of dengue (passive surveillance) were virologically confirmed. (ClinicalTrials.gov: NCT00875524).

Results: At baseline 139(77\%) were seropositive (titer $\geq 10 \mathrm{l} / \mathrm{dil}$ ) against dengue or Japanese encephalitis; $36 \%$ were seropositive to all four dengue serotypes. After the first CYD-TDV vaccination, $53 \%$ were seropositive to all four serotypes, increasing to $72 \%$ and $92 \%$ after the second and third vaccinations. In the control group seropositivity against all four dengue serotypes was $28 \%$ at baseline and slightly increased at $36 \%$ after the third injection 13 months later. After the third CYD-TDV vaccination, 96\% were seropositive to at least 3 serotypes, and geometric mean titers against DENV1-4 were respectively 129, 216, 169, and 146. Six serious adverse events (SAEs), unrelated to vaccination, were reported including 2 virologically-confirmed dengue cases after the second vaccination in the control group. Reactogenicity of CYD-TDV decreased after each vaccination, was slightly higher than placebo, but not higher than either active control.

Conclusions: Safety and reactogenicity of CYD-TDV were satisfactory and consistent with results from phase I and other phase II studies. Three doses of CYD-TDV induced a balanced neutralizing antibody response against the four dengue serotypes in children and adults living in a dengue endemic country.

Keywords: Dengue; Live attenuated tetravalent; CYD-TDV dengue vaccine; Recombinant vaccine; Safety; Immunogenicity; Phase II trial

\section{Introduction}

In recent decades dengue has expanded in tropical and subtropical areas around the world to become one of the most important vectorborne diseases of humans, with around 2.5 billion people living in regions where the disease is transmitted [1]. The overall annual burden of dengue as reported by World Health Organization (WHO) is an estimated 50-100 million dengue infections and 500,000 hospitalizations for severe form of the disease [1]. This burden is projected to continue to increase, notably in association with the geographic expansion of the mosquito vectors (i.e., Aedes aegypti and Aedes albopictus) [2].

It is a high public priority to develop a vaccine against dengue, specifically for highly endemic areas such as the Mekong delta, considering that no specific treatment is available, and considering the high cost and challenges around vector control programs to sustainably control disease [3]. The impact of dengue on patients and their families is important in terms of economics and quality of life, the health burden is considerable for endemic regions [4].

Several candidate vaccines against dengue are in clinical or preclinical development, one of which is currently being evaluated in clinical efficacy trials in Asia and Latin America [5]. This vaccine - the
CYD tetravalent dengue vaccine has been developed in compliance with WHO guidelines for the production and quality control of candidate tetravalent dengue vaccine [6], guidelines for the clinical evaluation of dengue vaccines in endemic areas [7], and guidelines for plaque reduction neutralization testing of human antibodies to dengue viruses [8].

This vaccine contains four recombinant, live, attenuated viruses (CYD-1-4) developed based on the attenuated yellow fever vaccine virus, YF-17D, from which the genes encoding for pre-membrane and

*Corresponding author: Tram Anh Wartel, Sanofi Pasteur Clinical R\&D, 87/2 CRC Tower 23rd Floor, All Seasons Place, Wireless Road, Lumpini, Pathumwan, Bangkok 10330, Thailand, Tel: (66)-2264-8813; Fax: (66)-2264-8800; E-mail: wartel-tram.anh@sanofipasteur.com

Received October 05, 2012; Accepted November 27, 2012; Published November 29, 2012

Citation: Tran NH, Luong CQ, Vu TQH, Forrat R, Lang J, et al. (2012) Safety and Immunogenicity of Recombinant, Live Attenuated Tetravalent Dengue Vaccine (CYD- TDV) in Healthy Vietnamese Adults and Children. J Vaccines Vaccin 3:162 doi:10.4172/2157-7560.1000162

Copyright: (c) 2012 Tran, et al. This is an open-access article distributed unde the terms of the Creative Commons Attribution License, which permits unrestricted use, distribution, and reproduction in any medium, provided the original author and source are credited. 
Citation: Tran NH, Luong CQ, Vu TQH, Forrat R, Lang J, et al. (2012) Safety and Immunogenicity of Recombinant, Live Attenuated Tetravalent Dengue Vaccine (CYD- TDV) in Healthy Vietnamese Adults and Children. J Vaccines Vaccin 3:162. doi:10.4172/2157-7560.1000162

Page 2 of 7

envelope proteins were removed and replaced with those of one of the four wild-type dengue viruses, one per serotype [5]. Phase 1 and early phase 2 trials have shown that a three-dose regimen of CYDTDV elicits a balanced neutralizing antibody response against all four serotypes in flavi virus-naïve populations, as well as in populations previously exposed to dengue. The safety and reactogenicity profile observed in these studies were considered to be promising, and provided reassurance to progress to larger scale trials [5].

Viet Nam has had amongst the highest dengue disease burden in the Asia Pacific region, with an estimated 1 million dengue cases and more than 2000 deaths during the period 1991-2004 [9]. The Mekong Delta area of Southern Viet Nam is particularly affected, with reported incidence rates among school-age children of 16.9-40.4 per 1000 person-years [10]. All four dengue serotypes have co-circulated in this area in recent years, usually with one serotype dominating at a given time [11,12]. This dominant serotype however changes periodically [9].

We report on the first clinical study of CYD-TDV in Viet Nam, conducted in an area where dengue is highly endemic. Dengue and Japanese encephalitis (JE) viruses are the primary vector-borne flaviviruses in the country, [13] and while JE vaccine is included in the national immunization program this had only been partially implemented in the South of Viet Nam at the time of the study.

\section{Methods}

\section{Trial design and participants}

This randomized, controlled, blind-observer, monocenter, phase II study took place in Long Xuyen city, An Giang province of Viet Nam. We recruited 180 individuals from four age cohorts (respectively 60, 60, 30 , and 30 aged $2-5,6-11,12-17$, and $18-45$ years), and randomized them in 2:1 ratio to receive CYD-TDV or control vaccines at Months 0,6 and 12 .

Individuals aged 2-45 years and in good health based on medical history, normal physical examination and laboratory parameters were eligible for inclusion. The main exclusion criteria were: personal or family history of thymic pathology, thymectomy or myasthenia; HIV, hepatitis B or C seropositivity tested at screening; immunodeficiency or receipt of immunosuppressive treatment; systemic hypersensitivity to any vaccine component; history of life threatening reaction to the trial vaccines or to vaccine containing any of the same substances; family history of allergy; vaccination with the control vaccines within the previous 3 years; pregnancy or breast-feeding. Females of child-bearing potential were instructed to use an effective means of contraception for at least 4 weeks prior to the first vaccination, until at least 4 weeks after the last vaccination.

Group allocation was performed using an Interactive Voice Response System (IVRS) based on randomization lists generated with the permuted block method and with age stratification. The study was conducted in accordance with Declaration of Helsinki and International Conference of Harmonization (ICH) guidelines for Good Clinical Practice (GCP) and was approved by the Vietnamese Ministry of Health Biomedical Research Ethics Committee as well as Joint Research Ethics Committee from Thailand. Written informed consent was obtained from all participants aged 12 years or older, and from the parent/guardian of all participants aged 2-17 years. Participants aged 8-11 signed an assent form. If either the participant or their parent/ legal representative were not able to read and sign the informed consent or assent form, it was signed by an impartial witness.
The study site fulfilled criteria set out in WHO guidelines for clinical evaluation of dengue vaccines in endemic areas [7].

\section{Vaccines}

The CYD vaccine is composed of $5 \pm 1 \log _{10}$ cell-culture infectious dose 50\% (CCID50) of each live, attenuated, dengue serotype 1,2,3,4 virus using $\mathrm{NaCl} 0.4 \%$ containing human serum albumin $2.5 \%$ as solvent. Control vaccines given at Months 0, 6 and 12 were, respectively: licensed meningococcal polysaccharide vaccine $\mathrm{A}+\mathrm{C}$ (Menomune- $\mathrm{A} /$ $\mathrm{C} / \mathrm{Y} / \mathrm{W}-135^{\circ}$, Sanofi Pasteur, France), saline placebo (the solvent used to reconstitute the CYD vaccine), and licensed typhoid VI polysaccharide vaccine (Typhim Vi ${ }^{\oplus}$, Sanofi Pasteur, France). Vaccines were prepared and administered in blind-observer conditions to prevent safety assessment bias.

\section{Safety and reactogenicity}

Participants were kept under observation for 30 minutes after each vaccination and were followed-up for safety until 6 months after the last vaccination. Participants or parents were provided with a thermometer, a ruler and a diary card to record any adverse event (AE) following each vaccination. The following AEs were documented and graded using a pre- defined severity scale as reported elsewhere solicited injection site reactions within 7 days (i.e., pain, erythema and swelling), solicited systemic reactions within 14 days (i.e., fever, headache, malaise, myalgia, and asthenia) and unsolicited AEs up to 28 days after each vaccination. All serious adverse events (SAE) were documented up to 6 months after the last vaccination.

\section{CYD-TDV vaccine viremia}

Cases of fever with body temperature $\geq 38^{\circ} \mathrm{C}$ for $\geq 48$ hours within 28 days after vaccination were assessed for CYD-TDV viremia by quantitative RT-PCR. Serology and viremia analyses were performed at the sponsor's global clinical immunology laboratory (dengue PRNT, RT-PCR; Swift water, PA) and at the Center for Vaccine Development (dengue ELISAs, JE PRNT; University of Mahidol, Thailand). All testing was performed under blind conditions.

\section{Serology}

In serum collected at screening, Japanese encephalitis virus neutralizing antibodies levels were assessed by a plaque reduction neutralization test with a 50\% endpoint (PRNT50) as described elsewhere $[13,14]$. Neutralizing serum antibody responses against dengue were measured using PRNT50 before and 28 days after each vaccination. Briefly, serial two-fold dilutions of heat-inactivated serum were mixed with a constant challenge dose of the dengue vaccine's four parental dengue wild-type viruses (target $80 \mathrm{PFU}$ per well for each serotype) and inoculated into wells of a 24-well plate of confluent Vero cells. The neutralizing antibody titer was calculated and expressed as the highest reciprocal serum dilution (1/dil) reducing the mean plaque count by $50 \%$ as compared with the mean virus plaque number obtained from the control wells. The lower limit of quantization (LLOQ) of the dengue PRNT50 was 10 (1/dil) and seropositivity rate expressed the percentage of subjects with seropositive samples.

\section{Passive detection and diagnosis of dengue fever}

In the event of a febrile episode with a body temperature of $\geq$ $38^{\circ} \mathrm{C}$ for $\geq 48$ hours and suspected dengue, participants/parents were instructed to return to the study center for laboratory diagnosis using quantitative dengue RT-PCR, and by commercial ELISA kits: NS1Ag ELISA (Platelia ${ }^{\mathrm{ax}}$, Biorad Laboratories, Marnes-La-Coquette, France), 
Citation: Tran NH, Luong CQ, Vu TQH, Forrat R, Lang J, et al. (2012) Safety and Immunogenicity of Recombinant, Live Attenuated Tetravalent Dengue Vaccine (CYD- TDV) in Healthy Vietnamese Adults and Children. J Vaccines Vaccin 3:162. doi:10.4172/2157-7560.1000162

Page 3 of 7

and Dengue IgM and IgG ELISA (EL1500M and EL1500G respectively, Focus Diagnostics Inc, CA, USA).

\section{Statistical method}

All the main analyses were descriptive. For the main parameters, 95\% Confidence Intervals of point estimates were calculated using the normal approximation for quantitative data and exact binomial distribution with Clopper-Pearson method for proportions. The sample size was arbitrarily set to 120 subjects in the dengue group and 60 subjects in the control group. Antibody titers were summarized by group and age cohort before and after each vaccination in terms of geometric mean titer (GMT), number and percentage with titer $\geq 10$ $1 /$ dil against each dengue serotype (seropositivity) and against at least one, two, three, or the four dengue serotypes. Analyses included all participants present at the first visit and having received at least one vaccination (i.e., safety analysis set [SAS] and full analysis set [FAS] with available results from blood taken after vaccination).

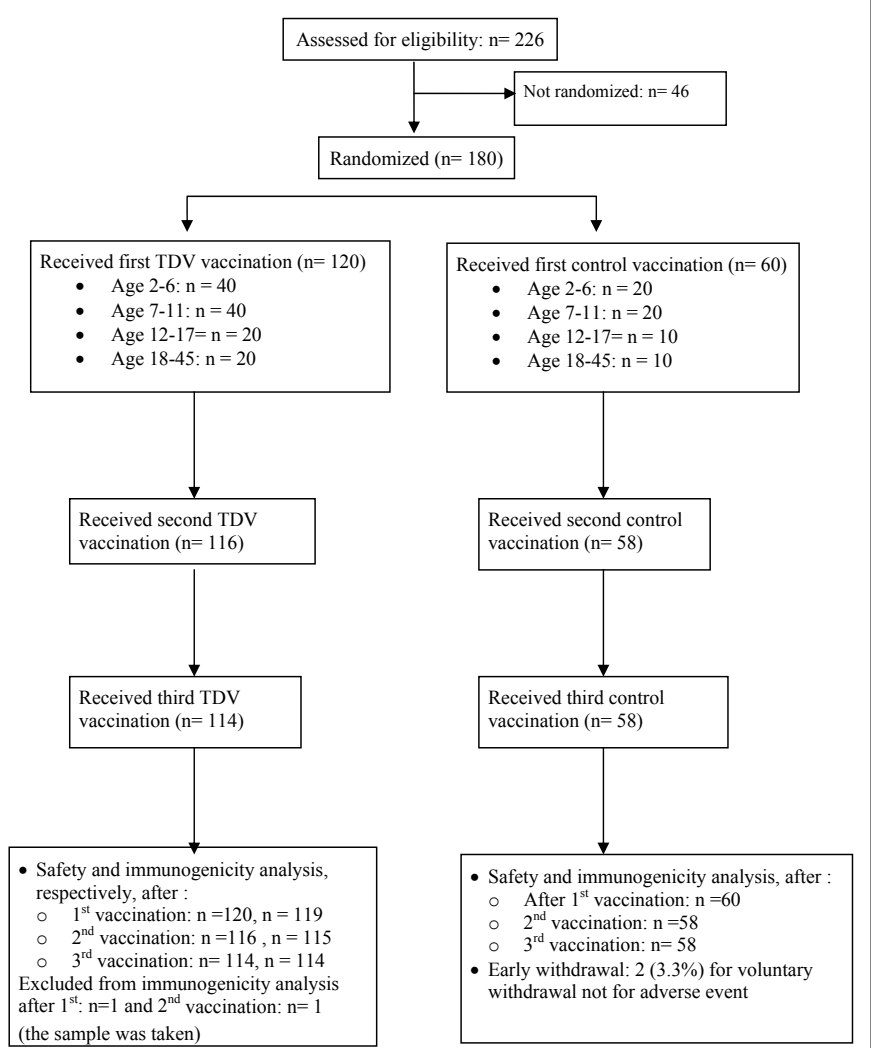

Figure 1: Participant flowchart.

\section{Results}

\section{Demographics and flavivirus immune status}

Between March and July 2009, we enrolled 180 participants (Figure $1)$ and vaccinated them with a first injection of CYD-TDV $(\mathrm{N}=120)$ or control vaccine $(\mathrm{N}=60) ; 172(96 \%)$ completed the study up to 6 month after the last vaccination. There were 6 early withdrawals in the dengue vaccine group and 2 in the control vaccine group. There were no discontinuations for safety reasons and none were lost to follow-up.

There were slightly more males enrolled in the control group $(58 \%$ males) than in the dengue vaccine group ( $48 \%$ males). The mean age, height, weight, and body mass index (BMI), were comparable between groups (Table 1). At baseline, a high proportion of $77 \%$ of participants (76\% in the dengue vaccine group and $80 \%$ in the control vaccine group) were seropositive against dengue or JE, and this proportion increased with age (Table 1). Almost $40 \%$ of participants had a baseline neutralizing antibody response against at least one dengue serotype: $39 \%(47 / 120)$ in the dengue vaccine group, $38 \%(23 / 60)$ in the control group. Seropositivity rates against dengue serotypes $1,2,3$, and 4 were, respectively: $25 \%, 18 \%, 53 \%$, and $23 \%$ among $2-5$ year-olds, $50 \%, 53 \%$, $55 \%$, and $43 \%$ among $6-11$ year-olds, $60 \%, 65 \%, 70 \%$, and $56 \%$ among 12-17 year-olds, and 95\%, 95\%, 95\%, and $85.0 \%$ among $18-45$ yearolds. Baseline seropositivity rates against the JE virus was similar in the dengue group (37\% [95\% CI: 28.3;46.3] and the control group (42\% [95\% CI: 29.1;55.1]).

\section{Safety and reactogenicity}

Table 2 shows the safety overview after any vaccination, by age cohort. Overall and by age group, solicited reactions were less frequently observed in the dengue vaccine group than in the control group. This was due to the solicited injection site reactions, reported for $33 \%$ [ $95 \% \mathrm{CI}: 24.2 ; 41.7$ ] for the dengue vaccine group than and $80 \%$ [95\%CI: 67.7; 89.2] in the control group. The reporting rates of solicited systemic reactions were similar between groups (Table 2). After dengue vaccination, reporting rates of solicited reactions and unsolicited adverse events appeared lower in children (i.e., $2-5$ yo and 6-11 yo) than in adolescents (i.e. 12-17 yo) or adults (18-45 yo). The reporting rates of unsolicited adverse events were similar between groups (Table 2). No adverse event led to study discontinuation, and there were no vaccine-related serious adverse events (SAE) (Table 2).

Injection site reactions after dengue vaccination were of grade 1 or 2 severity and resolved spontaneously, while a few grade 3 erythema and swelling reactions were recorded among 6-11 year-olds in the control group (Table 3). Most solicited injection site reactions after dengue vaccination were of grade 1 severity, and appeared within 3 days (data not shown). A few grade 3 systemic reactions (fever among 2-5 yearolds, and headache and asthenia among $18-45$ year-olds) were reported after dengue vaccination (Table 3 ).

\begin{tabular}{|c|c|c|c|c|c|c|c|c|c|c|}
\hline \multirow[b]{2}{*}{ Age cohort } & \multicolumn{5}{|c|}{ Dengue vaccine group } & \multicolumn{5}{|c|}{ Control group } \\
\hline & $2-5$ yrs & $6-11$ yrs & $12-17$ yrs & $18-45$ yrs & All & $2-5$ yrs & $6-11$ yrs & $12-17$ yrs & $18-45$ yrs & All \\
\hline $\mathrm{N}$ & 40 & 40 & 20 & 20 & 120 & 20 & 20 & 10 & 10 & 60 \\
\hline Male / n (\%) & $19(48)$ & $23(58)$ & $7(35)$ & $9(45)$ & $58(48)$ & $13(65)$ & $13(65)$ & $4(40)$ & $5(50)$ & $35(58)$ \\
\hline Mean age / years & 4.2 & 8.4 & 13.3 & 27.0 & & 4.6 & 9.0 & 13.4 & 29.9 & \\
\hline BMI / kg/m2 & 15.3 & 16.3 & 17.9 & 22.7 & & 14.5 & 16.5 & 17.8 & 21.4 & \\
\hline \multicolumn{11}{|c|}{ Baseline seropositivity / n (\%) } \\
\hline $\mathrm{JE}$ & $2(5)$ & $2(5)$ & $1(5)$ & $1(5)$ & $6(5)$ & $2(10)$ & $4(20)$ & $2(20)$ & $0(0)$ & $8(13)$ \\
\hline Dengue & $23(58)$ & $28(70)$ & $14(70)$ & $19(95)$ & $85(71)$ & $12(60)$ & $11(55)$ & $8(80)$ & $9(90)$ & $40(67)$ \\
\hline Dengue or JE & $26(65)$ & $30(75)$ & $15(75)$ & $20(100)$ & $91(76)$ & $14(70)$ & $15(75)$ & $10(100)$ & $9(90)$ & $48(80)$ \\
\hline
\end{tabular}

Table 1: Baseline demographic and flavivirus status characteristics of subjects. 
Citation: Tran NH, Luong CQ, Vu TQH, Forrat R, Lang J, et al. (2012) Safety and Immunogenicity of Recombinant, Live Attenuated Tetravalent Dengue Vaccine (CYD- TDV) in Healthy Vietnamese Adults and Children. J Vaccines Vaccin 3:162. doi:10.4172/2157-7560.1000162

Page 4 of 7

When considering reactogenicity specifically after each vaccination, solicited injection site reactions reported were more frequently after Meningococcal A+C vaccination (75\% [95\%CI: 62.1; 85.3]) than after the first dose of CYD-TDV (20\% [95\%CI: 13.3; 28.3]), and were more frequent after Typhoid fever vaccination (43\% [95\%CI: 30.2; 56.8]) than after the third dose of CYD-TDV (13\% [95\%CI: 7.6; 20.8]). The solicited reactions rate of the control vaccine was comparable to the dengue vaccine group for the second injection respectively $15.5 \%[95 \% \mathrm{CI}$ $7.2 ; 27.4]$ and $18.1 \%$ [95\%CI: $11.6 ; 26.3$. For the third vaccination, the solicited injection site reactions rate reported in the control group with

\begin{tabular}{|c|c|c|c|c|c|c|c|c|c|c|}
\hline \multicolumn{6}{|c|}{ Dengue vaccine group } & \multicolumn{5}{|c|}{ Control group } \\
\hline \multirow[t]{2}{*}{ Age cohort } & $2-5$ yrs & $6-11$ yrs & $12-17$ yrs & $18-45$ yrs & All & $2-5$ yrs & $6-11$ yrs & $12-17 \mathrm{yrs}$ & $18-45$ yrs & All \\
\hline & $\mathrm{N}=40$ & $\mathrm{~N}=40$ & $\mathrm{~N}=20$ & $\mathrm{~N}=20$ & $\mathrm{~N}=120$ & $\mathrm{~N}=20$ & $\mathrm{~N}=20$ & $\mathrm{~N}=10$ & $N=10$ & $\mathrm{~N}=60$ \\
\hline \multicolumn{11}{|c|}{ Subjects experiencing at least one/n, $\%(95 \% \mathrm{Cl})$} \\
\hline \multirow[t]{3}{*}{ Solicited reaction } & 17 & 25 & 17 & 15 & 74 & 16 & 19 & 9 & 8 & 52 \\
\hline & $43 \%$ & $63 \%$ & $85 \%$ & $75 \%$ & $62 \%$ & $80 \%$ & $95 \%$ & $90 \%$ & $80 \%$ & $87 \%$ \\
\hline & $(27.0 ; 59.1)$ & $(45.8 ; 77.3)$ & $(62.1 ; 96.8)$ & $(50.9 ; 91.3)$ & $(52.4 ; 70.4)$ & $(56.3 ; 94.3)$ & $(75.1 ; 99.9)$ & $(55.5 ; 99.7)$ & $(44.4 ; 97.5)$ & $(75.4 ; 94.1)$ \\
\hline Solicited injection & 6 & 14 & 10 & 9 & 39 & 13 & 19 & 9 & 7 & 48 \\
\hline \multirow[t]{2}{*}{ site reaction } & $15 \%$ & $35 \%$ & $50 \%$ & $45 \%$ & $33 \%$ & $65 \%$ & $95 \%$ & $90 \%$ & $70 \%$ & $80 \%$ \\
\hline & $(5.7 ; 29.8)$ & $(20.6 ; 51.7)$ & $(27.2 ; 72.8)$ & $(23.1 ; 68.5)$ & $(24.2 ; 41.7)$ & $(40.8 ; 84.6)$ & $(75.1 ; 99.9)$ & $(55.5 ; 99.7)$ & $(34.8 ; 93.3)$ & $(67.7 ; 89.2)$ \\
\hline Solicited systemic & 16 & 19 & 16 & 12 & 63 & 10 & 10 & 6 & 7 & 33 \\
\hline \multirow[t]{2}{*}{ reaction } & $40 \%$ & $48 \%$ & $80 \%$ & $60 \%$ & $53 \%$ & $50 \%$ & $50 \%$ & $60 \%$ & $70 \%$ & $55 \%$ \\
\hline & $(24.9 ; 56.7)$ & $(31.5 ; 63.9)$ & $(56.3 ; 94.3)$ & $(36.1 ; 80.9)$ & $(43.2 ; 61.7)$ & $(27.2 ; 72.8)$ & $(27.2 ; 72.8)$ & $(26.2 ; 87.8)$ & $(34.8 ; 93.3)$ & $(41.6 ; 67.9)$ \\
\hline \multirow[t]{3}{*}{ Unsolicited AE } & 8 & 7 & 11 & 7 & 33 & 5 & 6 & 5 & 4 & 20 \\
\hline & $20 \%$ & $18 \%$ & $55 \%$ & $35 \%$ & $28 \%$ & $25 \%$ & $30 \%$ & $50 \%$ & $40 \%$ & $33 \%$ \\
\hline & $(9.1 ; 35.6)$ & $(7.3 ; 32.8)$ & $(31.5 ; 76.9)$ & $(15.4 ; 59.2)$ & $(19.7 ; 36.4)$ & $(8.7 ; 49.1)$ & $(11.9 ; 54.3)$ & $(18.7 ; 81.3)$ & $(12.2 ; 73.8)$ & $(21.7 ; 46.7)$ \\
\hline \multirow[t]{3}{*}{ Unsolicited AR } & 0 & 1 & 2 & 0 & 3 & 0 & 0 & 0 & 0 & 0 \\
\hline & $0 \%$ & $3 \%$ & $10 \%$ & $0 \%$ & $3 \%$ & $0 \%$ & $0 \%$ & $0 \%$ & $0 \%$ & $0 \%$ \\
\hline & $(0 ; 8.8)$ & $(0.1 ; 13.2)$ & $(1.2 ; 31.7)$ & $(0 ; 16.8)$ & $(0.5 ; 7.1)$ & $(0 ; 16.8)$ & $(0 ; 16.8)$ & $(0 ; 30.8)$ & $(0 ; 30.8)$ & $(0 ; 6.0)$ \\
\hline \multirow[t]{3}{*}{ SAE } & 1 & 0 & 1 & 1 & 3 & 0 & 1 & 1 & 1 & 3 \\
\hline & $3 \%$ & $0 \%$ & $5 \%$ & $5 \%$ & $3 \%$ & $0 \%$ & $5 \%$ & $10 \%$ & $10 \%$ & $5 \%$ \\
\hline & $(0.1 ; 13.2)$ & $(0 ; 8.8)$ & $(0.1 ; 24.9)$ & $(0.1 ; 24.9)$ & $(0.5 ; 7.1)$ & $(0 ; 16.8)$ & $(0.1 ; 24.9)$ & $(0.3 ; 44.5)$ & $(0.3 ; 44.5)$ & $(1 ; 13.9)$ \\
\hline
\end{tabular}

- Solicited reaction: any adverse event (AE) pre-listed in the protocol that is automatically considered to be vaccine-related, and as such termed an

- 'adverse reaction' (AR)

- Solicited injection site reaction: any AR associated with the site of injection that is pre-listed in the protocol (pain, erythema, swelling)

- Solicited systemic reaction: any AR not associated with the site of injection that is pre-listed in the protocol (fever, headache, malaise, myalgia, asthenia)

- Unsolicited AE: any observed AE that is not a solicited injection site or solicited systemic reaction

- Unsolicited AR: any unsolicited AE that is considered to be vaccine-related

SAE: any untoward medical occurrence that at any dose results in death, is life-threatening, requires inpatient hospitalization or prolongation of existing hospitalization, results in persistent or significant disability/incapacity, is a congenital anomaly/birth defect, or is an important medical event Table 2: Safety overview after any vaccination by age cohort.

\begin{tabular}{|c|c|c|c|c|c|c|c|c|c|c|c|}
\hline \multicolumn{8}{|c|}{ Dengue vaccine group } & \multicolumn{4}{|c|}{ Control group } \\
\hline \multirow{2}{*}{\multicolumn{2}{|c|}{ Age cohort }} & $2-5$ yrs & $6-11$ yrs & $12-17$ yrs & $18-45$ yrs & All & $2-5$ yrs & $6-11$ yrs & $12-17$ yrs & $18-45$ yrs & All \\
\hline & & $\mathrm{N}=40$ & $N=40$ & $N=20$ & $\mathrm{~N}=20$ & $N=120$ & $N=20$ & $\mathrm{~N}=20$ & $\mathrm{~N}=10$ & $\mathrm{~N}=10$ & $\mathrm{~N}=60$ \\
\hline \multicolumn{12}{|c|}{ Subjects experiencing at least one / n, \% } \\
\hline \multicolumn{12}{|c|}{ Injection site reactions } \\
\hline Pain & Any & $5,13 \%$ & $12,30 \%$ & $10,50 \%$ & $7,35 \%$ & $34,29 \%$ & $13,65 \%$ & $19,95 \%$ & $9,90 \%$ & $7,70 \%$ & $48,80 \%$ \\
\hline \multirow[t]{2}{*}{ Erythema } & Any & $1,3 \%$ & $5,13 \%$ & $2,10 \%$ & 0 & $8,7 \%$ & $3,15 \%$ & $4,20 \%$ & $3,30 \%$ & $3,30 \%$ & $13,22 \%$ \\
\hline & Grade 3 & & & & & & & $1,5 \%$ & & & $1,2 \%$ \\
\hline \multirow[t]{2}{*}{ Swelling } & Any & 0 & $2,5 \%$ & $1,5 \%$ & $2,10 \%$ & $5,4 \%$ & $4,20 \%$ & $4,20 \%$ & 0 & $1,10 \%$ & $9,15 \%$ \\
\hline & Grade 3 & & & & & & & $1,5 \%$ & & & $1,2 \%$ \\
\hline \multicolumn{12}{|c|}{ Systemic reaction } \\
\hline \multirow[t]{2}{*}{ Fever } & Any & $12,30 \%$ & $10,25 \%$ & $9,45 \%$ & $2,10 \%$ & $33,28 \%$ & $6,30 \%$ & $5,25 \%$ & $2,20 \%$ & $3,30 \%$ & $16,27 \%$ \\
\hline & Grade 3 & $1,3 \%$ & & & & $1,1 \%$ & $2,10 \%$ & & & & $2,3 \%$ \\
\hline \multirow[t]{2}{*}{ Headache } & Any & $11,28 \%$ & $12,31 \% *$ & $10,50 \%$ & $9,45 \%$ & $42,35 \%$ & $5,25 \%$ & $7,35 \%$ & $5,50 \%$ & $1,10 \%$ & $18,30 \%$ \\
\hline & Grade 3 & & & & $1,5 \%$ & $1,1 \%$ & & & & & \\
\hline Malaise & Any & $7,18 \%$ & $9,23 \% *$ & $11,55 \%$ & $8,40 \%$ & $35,29 \%$ & $2,10 \%$ & $4,20 \%$ & $3,30 \%$ & $5,50 \%$ & $14,23 \%$ \\
\hline Myalgia & Any & $3,8 \%$ & $8,21 \% *$ & $8,40 \%$ & $5,25 \%$ & $24,20 \%$ & $3,15 \%$ & $3,15 \%$ & $5,50 \%$ & $3,30 \%$ & $14,23 \%$ \\
\hline \multirow[t]{2}{*}{ Asthenia } & Any & $3,8 \%$ & $6,15 \% *$ & $5,25 \%$ & $2,10 \%$ & $16,13 \%$ & $2,10 \%$ & 0 & 0 & $1,10 \%$ & $3,5 \%$ \\
\hline & Grade 3 & & $1,3 \%$ & & $1,5 \%$ & $2,2 \%$ & & & & & \\
\hline
\end{tabular}

${ }^{*} \mathrm{~N}=39$ due to missing data for one participant. Grade 3 injection site pain: Incapacitating, unable to perform usual activities, Grade 3 erythema or swelling: adults/ adolescents: $>10 \mathrm{~cm}$; children: $>5 \mathrm{~cm}$, Grade 3 fever $>39.0^{\circ} \mathrm{C}$, Grade 3: headache, malaise, myalgia, or asthenia: Daily activity prevented

Table 3: Percentages of individuals (by age and vaccine group) reporting at least 1 injection site and/or systemic reaction after any vaccination (safety analysis set). 
Citation: Tran NH, Luong CQ, Vu TQH, Forrat R, Lang J, et al. (2012) Safety and Immunogenicity of Recombinant, Live Attenuated Tetravalent Dengue Vaccine (CYD- TDV) in Healthy Vietnamese Adults and Children. J Vaccines Vaccin 3:162. doi:10.4172/2157-7560.1000162

Page 5 of 7

Typhoid fever vaccine was higher $(43.1 \%$ [95\% CI:30.2; 56.8] than in the dengue vaccine group (13.2\% [95\%CI: 7.6; 20.8]) (data not shown).

Solicited systemic reactions rates diminished after each successive dose of the dengue vaccine, and were similar in the control and dengue vaccine groups; respectively $48 \%$ (95\%CI: $35.2 ; 61.6)$ and $42 \%(95 \% \mathrm{CI}$ : $32.7 ; 51.0)$ after the first dose, $17 \%(95 \% \mathrm{CI}: 8.6 ; 29.4)$ and $27.6 \%$ [95\%CI: 19.7; 36.7]) after the second dose, and 24\% (95\%CI: 13.9 ; $37.2)$ and $15 \%(95 \% \mathrm{CI}: 8.9 ; 22.8)$ after the third. Overall, after any vaccination, headache (35\%) was the most frequently reported solicited systemic reaction in the dengue group followed by malaise (29\%), fever (28\%), myalgia (20\%) and asthenia (13\%) while in the control group, the following reactions were reported: headache $(30 \%)$, fever $(27 \%)$, malaise (23\%), myalgia (23\%) and asthenia (5\%). A summary of safety results according to age group is shown in table 3.

\section{Serious adverse events}

There were six serious adverse events (SAEs) due to hospitalization, none of which were related to vaccination. Three occurred in the dengue group $(3 / 120,2.5 \%)$, and three occurred in the control group $(3 / 60$, $5 \%)$. The three SAEs in the dengue group were acute gastritis, acute Pharyngitis, and dengue hemorrhagic fever (DHF) grade 1 meeting the 1997 WHO case definition [15]. The three SAEs in the control group were three cases of dengue infection ( 2 cases of DHF grade 1 and 1 case of DHF grade 2).

\section{Fever and symptomatic dengue cases}

From the start of the study through the period of 6 months followup after the last vaccination, 7 clinically diagnosed dengue cases were
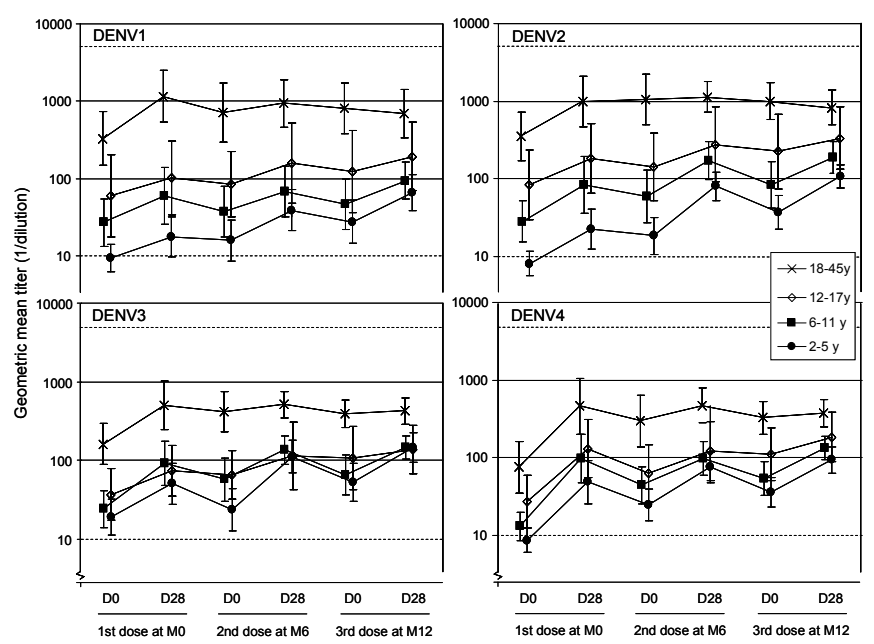

Figure 2: Dengue serotype-specific neutralizing antibody GMTs before (D0) and 28 days (D28) after 3 doses of CYD-TDV in 4 age groups. reported, 4 in the dengue vaccine group and 3 in the control vaccine group. All patients recovered without sequela. Of the 7 suspected dengue cases, 4 cases were inpatient cases ( 3 in the control vaccine group, 1 in the dengue vaccine group) and 3 outpatient cases (all in the dengue vaccine group). The outpatient case in the dengue vaccine group occurred within 28 days after the second vaccination in an 8 year-old female with a concomitant diagnosis of tonsillitis. Dengue infection was not confirmed in virological or serological tests. As this case occurred within 28 days of a dengue vaccine, serum was tested for vaccine viremia and found negative. Neither of the 2 outpatient cases in the control group were confirmed as dengue by serological or virological testing, and tonsillitis or another viral infection were suspected in addition to dengue. In the control group, there were 3 dengue cases that required hospitalization (i.e., one virologically confirmed grade I DHF due to serotype 1, one virologically confirmed grade II DHF also due to serotype 1, and one serologically probable grade I DHF). In the dengue vaccine group, there was one hospitalized grade I DHF in an 18 year-old male subject that occurred 93 days after the first vaccination. Virological tests for dengue were negative, although anti-dengue IgM was detected in acute and convalescent samples. He was seropositive for both dengue and JE viruses at baseline and on Day 28 after the first vaccination, his neutralizing antibody levels against serotypes 1-4 were 106, 191, 86, and 169 respectively. Of the 7 suspected dengue cases, dengue infection was therefore virologically confirmed in 2 cases in the control group $(\mathrm{N}=60)$, and none in the dengue vaccine group $(\mathrm{N}=120)$.

\section{Immunogenicity}

Antibody responses increased after each of the three doses of dengue vaccines, CYD-TDV against each serotype for all four serotypes in all age cohorts (Figure 2). On Day 28 after the complete 3-dose CYD-TDV schedule, GMTs against serotypes 1-4 were respectively 129, 216, 169, and $146,92 \%$ of vaccines were seropositive to all 4 dengue serotypes, compared with $36.2 \%$ in the control group (Table 4 ). Antibody responses after vaccination appeared to increase with increasing age with GMTs after the third CYD-TDV dose in the range 64.7-143 among 2-5 yearsold, 93.9-185 among 6-11 years-old, 135-334 among 12-17 years-old, and 375-825 among adults (Figure 2).

Antibody responses to CYD-TDV were higher among those who were flavivirus seropositive at baseline compared with those who were flavivirus seronegative (Figure 3). In the flavivirus positive subgroup, GMTs increased to values in the range 187-316 after 3 doses of CYDTDV, at which point $97.7 \%$ were seropositive against all four serotypes. In those who were flavivirus negative, GMTs increased to values in the range $31.2-75.5$, at which point $75 \%$ were seropositive against all four serotypes.

\section{Discussion}

This is the first clinical trial of a dengue vaccine candidate in

\begin{tabular}{|c|c|c|c|c|c|c|c|c|}
\hline & \multirow[b]{2}{*}{ DENV1 } & \multicolumn{2}{|c|}{ Dengue Group ( $\mathrm{N}=120)$} & \multirow[b]{2}{*}{ DENV4 } & \multirow[b]{2}{*}{ DENV1 } & \multicolumn{2}{|c|}{ Control Group ( $\mathrm{N}=60)$} & \multirow[b]{2}{*}{ DENV4 } \\
\hline & & DENV2 & DENV3 & & & DENV2 & DENV3 & \\
\hline \multicolumn{9}{|c|}{ Geometric mean titer } \\
\hline Baseline & 32.8 & 33.7 & 32.5 & 17.1 & 19.6 & 27.2 & 20.5 & 13.9 \\
\hline $95 \% \mathrm{Cl}$ & $21.7 ; 49.5$ & $23.0 ; 49.6$ & $23.7 ; 44.6$ & $12.9 ; 22.6$ & $12.0 ; 31.8$ & $15.3 ; 48.1$ & $13.2 ; 31.9$ & $9.28 ; 20.9$ \\
\hline After 3rd dose & 129 & 216 & 169 & 146 & 25.3 & 30.4 & 25.2 & 17.4 \\
\hline $95 \% \mathrm{Cl}$ & $0.5 ; 183$ & $163 ; 286$ & $134 ; 214$ & $115 ; 184$ & $13.7 ; 46.8$ & $16.7 ; 55.1$ & $16.3 ; 39.1$ & $11.2 ; 27.0$ \\
\hline
\end{tabular}

Table 4: Dengue serotype-specific antibody response before first dose and 28 days after third dose of CYD-TDV or control vaccine. 


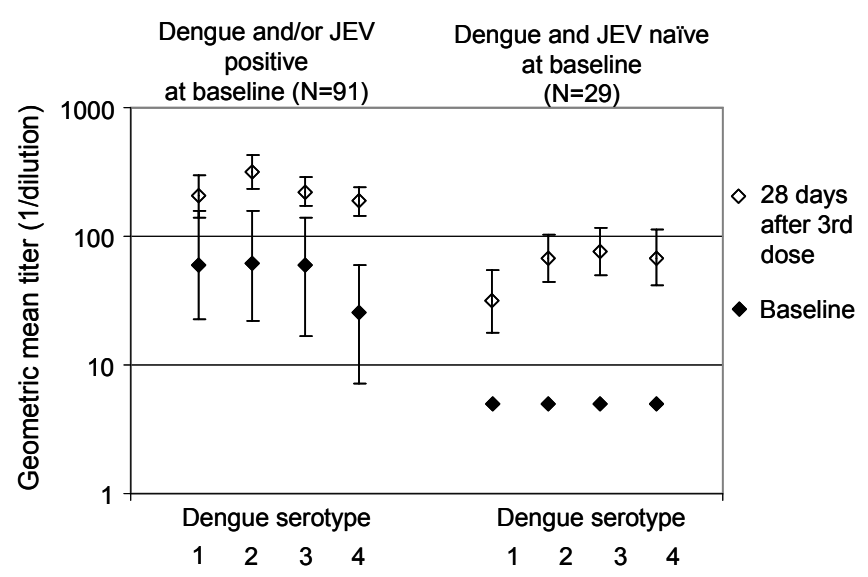

Figure 3: Effect of baseline flavivirus immune status on the dengue serotypespecific neutralizing antibody GMT at baseline and 28 days after a third dose of CYD-TDV.

Viet Nam. The high and age- dependent dengue seropositivity levels observed at the start of the study confirm the high endemicity of the disease in Long Xuyen. Regardless of flavivirus serostatus at baseline, antibody responses against the four dengue serotypes increased after each vaccination to levels consistent to those observed in other studies with this vaccine candidate in Philippines and Thailand, where dengue is also endemic [16,17]. That neutralizing antibody levels after the third dengue vaccination were higher in individuals with pre-existing antibodies against dengue or JE compared to those who were seronegative, is suggestive of a role of pre-existing flavivirus antibodies on dengue vaccine-induced antibody responses. Similar to results reported from the Philippines, immune responses to dengue vaccination also varied with age [16]. Post-vaccination antibody titers were highest in adults, whereas a higher relative increase compared to baseline was seen in younger age groups. In Singapore, where dengue is also endemic, but at lower levels than in Viet Nam, immune responses to the CYD- TDV vaccine candidate were also seen to vary with age, but with lower GMTs than those observed in our study (range 43-100) [18]. A similar effect of pre-existing flaviviral immune responses on the antibody responses after dengue vaccination has been observed with pre- existing yellow fever antibody responses $[19,20]$.

CYD-TDV was immunogenic in this cohort of 2-45 year-olds and elicited balanced immune response against all four serotypes, as observed previously $[16,18]$. However the exact relationship between antibody titers, as assessed by PRNT50, and protection against dengue is not known, and a phase IIb proof-of-concept efficacy study of CYD-TDV conducted in Ratchaburi, Thailand showed protection against dengue serotypes 1,3 , and 4 , but not against the serotype 2 viruses circulating at the time, despite having elicited satisfactory PRNT50 antibody titers against all four serotypes at levels comparable to those observed in our study [17]. The safety profile of the dengue vaccine candidate in this study was good, with no immediate unsolicited AEs, withdrawals for safety reasons, vaccine-related SAEs, or any other safety issues. Overall reactogenicity appeared similar to that of two licensed control vaccines (i.e., Meningococcal Polysaccharide Vaccine $\mathrm{A}+\mathrm{C}$, and Typhoid Vi polysaccharide vaccine (Typhim $\mathrm{Vi}^{\circ}$ ) and the rate of solicited reactions was lower in the CYD-TDV group. Local and systemic reactions were mild to moderate and short-lived and injection site pain was the most frequently reported injection site reaction. Reactogenicity was lower after the second and third vaccinations, compared to that documented after the first, confirming the good tolerability of this vaccine candidate.

Seven cases of dengue were clinically-suspected, but only two cases, both in the control group, were virologically confirmed to be dengue. Other cases tested positive for anti-dengue IgM, but given the probable effect of CYD-TDV vaccination on IgM and IgG responses, any such cases occurring after dengue vaccination (one case in our study) cannot conclusively be considered as dengue. The investigator's reporting of a second clinical diagnosis in these suspected, but not virologicallyconfirmed cases, adds to the likelihood that these cases were not dengue. The significant discordance between clinical diagnosis and laboratory confirmation highlights the importance of laboratory diagnosis of suspected infections.

CYD-TDV is a live attenuated viral vaccine and viral replication is expected to occur. Previous studies have shown that after vaccination, low levels of the CYD viruses can be detected in serum in a minority of vaccines between 7-14 days after vaccinations, but this has not been associated with any safety issues [16,22]. Here, one suspected dengue case occurring within 28 days of a dengue vaccination was tested and found negative for dengue vaccine viremia.

In summary, this study conducted in an area at high risk for dengue infection showed that a 3 dose CYD-TDV regimen elicited balanced neutralizing antibody responses against all four dengue virus serotypes in a wide range of age group, with a satisfactory safety profile comparable to that observed in previous trials. These data support the continued clinical development of the CYD-TDV vaccine candidate.

\section{Conflict of Interest}

TNH was the study principal investigator, LCQ and VTQH were coinvestigators. They did not receive direct payment from the study sponsor for their contributions and declare no conflicts of interest. WTA and FR designed the study. WTA, LJ, VQD, and BA interpreted the study results and are Sanofi Pasteur employees except VQD at the time of manuscript finalization.

\section{Financial Disclosure}

The study was sponsored by Sanofi Pasteur.

\section{Acknowledgments}

The authors would like to thank all the participants and their parents/guardians who participated in the study, Dr. Nguyen Chi Cong, Long Xuyen Hospital Director and investigator, Dr. Phan Kim Hoang, Vice-Director of An Giang Preventive Medicine Center and site coordinator, and the study site personnel, Dr. Simonetta Viviani, Mark Boaz, and Grenville Marsh at Sanofi Pasteur France and US for comments and suggestions on the drafts of this manuscript, Tran Phuong Thuy and Nuchra Sirisuphmitr at Sanofi Pasteur Asia, and Dr. Sutee Yoksan at the Center for Vaccine Development, Mahidol University in Thailand for their valuable contributions to the study.

\section{Trial Registration}

The trial is registered on ClinicalTrials.gov (National Clinical Trials Identifier NCT00875524)

\section{References}

1. World Health Organization (2012) Dengue and severe dengue, Fact sheet No117.

2. World Health Organization Regional Office for South-East Asia (2011) Comprehensive Guidelines for Prevention and Control of Dengue and Dengue Haemorrhagic Fever.

3. Guzman A, Isturiz RE (2010) Update on the global spread of dengue. Int $J$ Antimicrob Agents 36: 40-42.

4. Lam SK, Burke D, Capeding MR, Chong CK, Coudeville L, et al. (2011) Preparing for introduction of a dengue vaccine: Recommendations from the $1 \mathrm{st}$ Dengue v2V Asia-Pacific Meeting. Vaccine 28: 9417-9422.

5. Guy B, Barrere B, Malinowski C, Saville M, Teyssou R, et al. (2011) From 
Citation: Tran NH, Luong CQ, Vu TQH, Forrat R, Lang J, et al. (2012) Safety and Immunogenicity of Recombinant, Live Attenuated Tetravalent Dengue Vaccine (CYD- TDV) in Healthy Vietnamese Adults and Children. J Vaccines Vaccin 3:162. doi:10.4172/2157-7560.1000162

Page 7 of 7

research to phase III: preclinical, industrial and clinical development of the Sanofi Pasteur tetravalent dengue vaccine. Vaccine 29: 7229-7241.

6. World Health Organization (2003) WHO TRS 932 Guideline for the production and quality control of candidate tetravalent dengue vaccines.

7. World Health Organization (2008) Guidelines for the clinical evaluation of dengue vaccine in endemic areas, WHO/IVB/08.12.

8. World Health Organization (2007) Guidelines for plaque reduction neutralization testing of human antibodies to dengue viruses, WHO/IVB/07.07.

9. World Health Organization. Regional Office for South-East Asia and for the Western Pacific, Health in Asia and the Pacific 2008, New Delhi, India. WHO Regional Office for South-East Asia: 539.

10. Tien NT, Luxemburger C, Toan NT, Pollissard-Gadroy L, Huong VT, et al. (2010) A prospective cohort study of dengue infection in schoolchildren in Long Xuyen, Viet Nam. Trans R Soc Trop Med Hyg 104: 592-600.

11. Ha DQ, Tien NT, Huong VT, Loan HT, Thang CM (1998) Dengue epidemic in southern Vietnam. Emerg Infect Dis 6: 422-425.

12. Vu TT, Holmes EC, Duong V, Nguyen TQ, Tran TH, et al. (2010) Emergence of the Asian 1 genotype of dengue virus serotype 2 in Vietnam: in vivo fitness advantage and lineage replacement in South-East Asia. PLoS Negl Trop Dis 4: 757.

13. Yen NT, Duffy MR, Hong NM, Hien NT, Fischer M, et al. (2010) Surveillance for Japanese encephalitis in Vietnam, 1998-2007. Am J Trop Med Hyg 83: 816-819.

14. Nasveld PE, Ebringer A, Elmes N, Bennett S, Yoksan S, et al. (2010) Long term immunity to live attenuated Japanese encephalitis chimeric virus vaccine: randomized, double-blind, 5-year phase II study in healthy adults. Hum Vaccin 6: 1038-1046.
15. World Health Organization (1997) Dengue Haemorrhagic Fever, Diagnosis Treatment, Prevention and Control (2ndedn), WHO, Geneva.

16. Capeding RZ, Luna IA, Bomasang E, Lupisan S, Lang J, et al. (2011) Liveattenuated, tetravalent dengue vaccine in children, adolescents and adults in a dengue endemic country: randomized controlled phase I trial in the Philippines. Vaccine 29: 3863-3872.

17. Sabchareon A, Wallace D, Sirivichayakul C, Limkittikul K, Chanthavanich P, et al. (2012) Protective efficacy of the recombinant, live-attenuated, CYD tetravalent dengue vaccine in Thai schoolchildren: a randomised, controlled phase $2 \mathrm{~b}$ trial. Lancet 380: 1559-1567.

18. Sin Leo Y, Wilder-Smith A, Archuleta S, Shek L, Chong CY, et al. (2012) Immunogenicity and safety of recombinant tatravalent dengue vaccine (CYDTDV) in individuals aged 2-45 y. Hum Vaccin Immunother 8.

19. Lanata CF, Andrade T, Gil Al, Terrones C, Valladolid O, et al. (2012) Immunogenicity and safety of tetravalent dengue vaccine in 2-11 year-olds previously vaccinated against yellow fever: Randomized, controlled, phase II study in Piura, Peru. Vaccine 30: 5935- 5941.

20. Poo J, Galan F, Forrat R, Zambrano B, Lang J, et al. (2010) Live-attenuated Tetravalent Dengue Vaccine in Dengue-naive Children, Adolescents, and Adults in Mexico City: Randomized Controlled Phase 1 Trial of Safety and Immunogenicity. Pediatr Infect Dis J.

21. Morrison D, Legg TJ, Billings CW, Forrat R, Yoksan S, et al. (2010) A nove tetravalent dengue vaccine is well tolerated and immunogenic against all 4 serotypes in flavivirusnaive adults. J Infect Dis 201: 370-377.

22. Guy B, Saville M, Lang J (2010) Development of Sanofi Pasteur tetravalent dengue vaccine. Hum Vaccin 6 . 